\title{
Evaluation of a Low-Cost Insulin Infusion Pump Prototype: in Vitro Preliminary Results
}

\author{
Tenorio FS ${ }^{1}$, Martins LEG ${ }^{2 *}$, Cunha TS $^{2 *}$ \\ ${ }^{1}$ Federal University of São Paulo \\ Department of Medicine, São Paulo, Brazil \\ Fernanda.tenorio@unifesp.br; legmartins@unifesp.br \\ ${ }^{2}$ Federal University of São Paulo \\ Institute of Science and Technology, São José dos Campos, Brazil \\ ts.cunha@unifesp.br \\ *both last authors equally contributed to this work
}

\begin{abstract}
Diabetes Mellitus (DM) is considered a worldwide epidemic, posing a major challenge for health systems around the world. According to the World Health Organization (WHO), 1 in 11 people lives with diabetes, indicating that there are around 684 million of diabetic patients in the world. According to data from the Brazilian Society of Diabetes, the estimated number of children and adolescents affected by DM type 1 (DM1) in Brazil is approximately 100 thousand, and the majority of them are individuals under 14 years old. DM1 is generally associated with extremely serious acute and chronic complications, due to improper blood glucose control. One of the therapies used for DM treatment is the insulin infusion pump (IIP), and although approximately 15\% of DM1 patients have an absolute indication for its use, the number of patients with access to this type of treatment is still very low, especially in Brazil. The main limiting factor for the use of IIP in Brazil is due to the high cost of the device (around US\$3,750 or R $\$ 15,000$ ) and supplies such as syringes, batteries, cannulas and catheters (US $\$ 250.00 /$ month or $\mathrm{R} \$ 1,000 /$ month), since these are imported items. These factors restrict the use of IPP by the majority of DM1 patients. Our main goal is to develop a low-cost commercial IIP whose estimated value would represent onequarter of the value of available IIP commercialized in Brazil. We are currently developing and testing (in vitro) this low-cost IIP prototype, which works using cheaper solutions, including a commercial battery and also a universal syringe. Considering that we are working with a critical medical system, the aim of this study was to perform in vitro quantitative tests to evaluate the accuracy of the low-cost IIP prototype, and based on the results, adapt our system according to the performance. Based on preliminary in vitro analysis, it was possible to conclude that the prototype presents precision, as determined by the absence of statistical differences during the tests. This gives important support for further analysis of the prototype and improvement of the system.
\end{abstract}

Keywords: Diabetes Mellitus, Hyperglicemia, Low-Cost Insulin Infusion Pump, Mechanical System, Embedded System.

\section{Introduction}

Diabetes Mellitus (DM) is considered a worldwide epidemic, and according to World Health Organization (WHO), 1 in 11 people lives with diabetes, indicating that there are around 684 million of diabetic patients in the world. In Brazil, 14 million people (6.8\% of the population) live with DM, and this number continues to grow. DM is one of the four major types of noncommunicable diseases, characterized by chronic hyperglycemia due to failure in secretion, action, or both of insulin[1]. When blood glucose is improperly managed, DM can lead to serious macro and microvascular complications, which burden health services [2] and implicate in high medical, economic and social costs. World spending with the disease reaches $\$ 327$ billion [3] and in 2018, around \$51 billion ( $\mathrm{R} \$ 203$ billion) were spent with the disease [4].

DM type 1(DM1) accounts for 5 to $10 \%$ of all DM cases [5] and is usually diagnosed before 15 years of age [6], [7], indicating that DM1 patients will cope with the disease for most of their lives. In Brazil, approximately 100,000 people under 14 years old live with DM1 [8] and only 15\% of them have well-controlled glycemia [6,7]. As mentioned before, uncontrolled blood glucose increases the risk for the development of serious chronic complications, including nephropathy, neuropathy, retinopathy and cardiomyopathy [9]. Therefore, current data suggest that the costs with the disease tend to increase in the next years unless the public system provides DM patients access to treatments associated with better glycemic control.

Among the available treatments and technologies for DM management, the use of insulin infusion pump (IIP) patients has been shown to improve glycemic control of DM1, reduce severe hyperglycemia and the number of hospitalizations due to diabetic ketoacidosis, compared to conventional treatment with injections [10].. Although the benefits from the use of IPP 
are expressive, and approximately 15\% of DM1 patients have an absolute indication for its use, the number of diabetics with access to this type of treatment is still very low, especially in Brazil. The main limiting factor for the use of IIP in Brazil refers to the high cost of the device (around US $\$ 3,750$ or R $\$ 15,000$ ) and supplies such as syringes, batteries, cannulas and catheters (US\$250.00 / month or R $\$ 1,000 /$ month), since these are imported items, restricting the use of IPP by the majority of DM1 patients.

In the view of the problems reported, our research group in Brazil, in cooperation with the Brazilian company DeltaLife [11], has been working on the development of a low-cost IIP prototype during the last 5 years [12], [13]. Our main goal is to develop a low-cost commercial IIP whose estimated value would represent one quarter of value of available IIP commercialized in Brazil. We are currently developing and testing (in vitro) the mechanical system of this low-cost IIP prototype, which works using cheaper solutions, including a commercial battery and also a universal syringe, previously described in detail [12]. In brief, our prototype constitutes a device controlled by embedded computer systems, which injects continuously into the subcutaneous tissue, micro-doses of insulin during 24 hours of the day, simulating the pancreatic secretion of this hormone.

Considering that we are working with a critical medical system, our aim at this stage is to perform in vitro quantitative tests to evaluate the accuracy of the low-cost IIP prototype, and based on the results, adapt our system according to the performance.

\section{Infusion System}

The IIP is a device controlled by embedded computer systems, which inject microdoses of insulin continuously over a pre-programmed time interval. Its operation is described in Fig 1, showing that a microcontroller controls the motor and the mechanical system acts on a syringe containing insulin, infusing micro-doses of this hormone into the subcutaneous tissue of the patient.

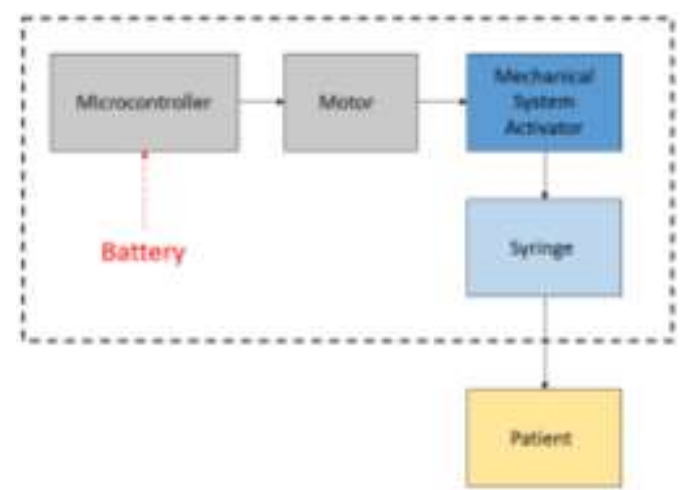

Fig. 1: System Diagram of the low-cost Insulin Infusion Pump prototype.

\section{Methodology}

The methodology was based on the combination of two analyses that aim to evaluate in vitro the precision of the low-cost IIP prototype. Below, we describe how the assays were performed and its subsequent statistical analyses.

\subsection{Experimental Design}

The in vitro protocol consisted of 10 complete tests ( $\mathrm{n}=10$ tests) performed during $2 \mathrm{~h}$. During the tests, the lowcost IIP prototype was programmed to infuse continuously a fixed dose of the fluid, every 5 minutes (24 measurements/test). When a 5-minute cycle was complete, the observer registered the weight corresponding to the volume of fluid infused by the syringe. At the end of $2 \mathrm{~h}, 24$ samples (measurements) were taken and the device was shut down and restarted. The procedure was repeated in controlled environment $\left(\mathrm{T}=25 \pm 2{ }^{\circ} \mathrm{C}\right.$, humidity $\left.=60 \pm 5 \%\right)$. After that, statistical analyses were performed comparing the data of each test and between the tests.

\subsubsection{Precision and Statistical Analysis}

The experimental tests were performed to analyze the precision of the low-cost IIP prototype, comparing the differences within each test (intra-test), and among the 10 tests (inter-tests). In this way, the fluid ejected by the syringe, 
after each five-minute cycle, was immediately weighed on a high-precision analytical balance (Shimadzu AUY220, Japan). The data were stored in a database and for further analyses. The precisio

$\mathrm{n}$ of the low-cost IIP prototype was statistically analyzed by one-way ANOVA, using MATLAB® 2015a. In this study, we considered $\alpha$ equal to 0.05 .

\section{Results and Discussion}

The results regarding the precision of the prototype are presented in Figure 2 and Table 1. Data shows that there is no statistical difference neither among the tests, neither within the tests. The $\mathrm{p}$ value obtained after the statistical analysis indicates that the results are extremely similar $(\mathrm{p}=0.99)$, as shown in Table 4.

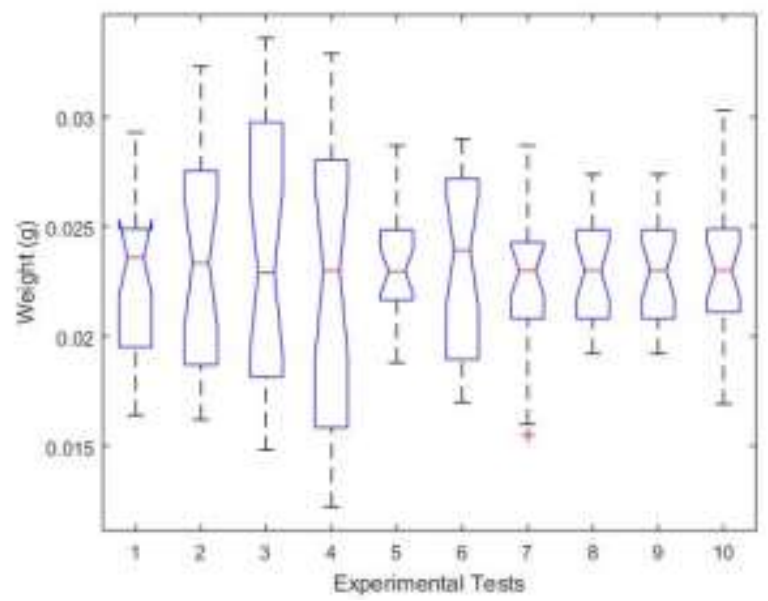

Fig. 2: Representative boxplot of the data obtained in experimental tests $(n=10)$. Each bar represents the results from the 24 infusions performed in each test. The horizontal red line inside the box indicates the median value within each test and the upper and lower edges of the box indicate the quartiles; the vertical bars indicate the range and the red dots are outliers of the corresponding groups.

Significance was determined by one-way ANOVA.

Table 1: Data obtained in experimental tests $(\mathrm{n}=10)$, presented as weight $(\mathrm{g})$, confidence intervals and errors.

\begin{tabular}{|c|c|c|c|}
\hline Group & Weight $^{1}(\mathrm{~g})$ & CI $(95 \%)$ & Error \\
\hline Test 01 & $0.0228 \pm 0.0028$ & {$[0.0217 ; 0.0240]$} & 0.0012 \\
\hline Test 02 & $0.0232 \pm 0.0039$ & {$[0.0150 ; 0.0249]$} & 0.0017 \\
\hline Test 03 & $0.0236 \pm 0.0057$ & {$[0.0213 ; 0.0261]$} & 0.0024 \\
\hline Test 04 & $0.0228 \pm 0.0057$ & {$[0.0205 ; 0.0252]$} & 0.0024 \\
\hline Test 05 & $0.0232 \pm 0.0021$ & {$[0.0223 ; 0.0242]$} & 0.0009 \\
\hline Test 06 & $0.0232 \pm 0.0036$ & {$[0.0217 ; 0.0247]$} & 0.0015 \\
\hline Test 07 & $0.0225 \pm 0.0026$ & {$[0.0214 ; 0.0237]$} & 0.0011 \\
\hline Test 08 & $0.0227 \pm 0.0019$ & {$[0.0219 ; 0.0236]$} & 0.0008 \\
\hline Test 09 & $0.0227 \pm 0.0019$ & {$[0.0219 ; 0.0236]$} & 0.0008 \\
\hline Test 10 & $0.0231 \pm 0.0025$ & {$[0.0221 ; 0.0242]$} & 0.0011 \\
\hline
\end{tabular}

${ }^{1}$ Data are presented as Mean \pm SD (Standard Deviation). CI represents the confidence interval of them and their respective errors.

Table 2: Data regarding one-way ANOVA, performed to evaluate the intra-group and inter-group differences.

\begin{tabular}{|c|cccccc|}
\hline Variation Source & SS & df & MS & F & P Value & Critical F \\
\cline { 1 - 5 } Between Groups & 0.853 & 9 & 0.0947 & 0.07 & 0.9999 & 1.9207 \\
\cline { 1 - 5 } Within the Groups & 328.012 & 230 & 1.4261 & & & \\
\cline { 1 - 5 } Total & 328.865 & 239 & & & & \\
\hline
\end{tabular}


SS is the sum of squares and df is the degrees of freedom. The total degrees of freedom are the numbers of total infusion samples minus one, which is $240-1=239$. The degrees of freedom among the groups are expressed as the number of experimental tests performed minus one $(10-1=9)$. The degrees of freedom within the group are expressed as the total degrees of freedom minus the degrees of freedom among groups $(239-9=230)$. MS is the mean square error, given by SS / df of each source of variation. The $\mathrm{F}$ statistic is the ratio of the mean squared errors. $\mathrm{p}=0,99$.

\section{Conclusion}

Based on the preliminary in vitro analysis, it was possible to conclude that the prototype presents precision, considering the obtained $\mathrm{p}$ value $(\mathrm{p}=0.99)$, indicating therefore that there is no statistical difference among the tests, nor within the tests. This gives important support for further analysis of the prototype and improvement of the system.

\section{Acknowledgements}

This work was funded by the Coordenação de Aperfeiçoamento de Pessoal de Nível Superior - Brasil (CAPES, Finance Code 001) and FAPESP (2010/511904-9).

\section{References}

[1] D. Of and D. Mellitus, "Diagnosis and Classi fi cation of Diabetes Mellitus," vol. 37, no. January, pp. 81-90, 2014.

[2] "The Cost of Diabetes: American Diabetes Association ${ }^{\circledR} . " \quad[O n l i n e] . \quad$ Available: http://www.diabetes.org/advocacy/news-events/cost-of-diabetes.html. [Accessed: 24-May-2019].

[3] "Qual a situação da diabetes no Brasil?: SBAC." [Online]. Available: http://www.sbac.org.br/noticias/qual-asituacao-da-diabetes-no-brasil/. [Accessed: 24-May-2019].

[4] J. E. P. de Oliveira, R. M. M. Júnior, and S. Vencio, Diretrizes 2017-2018. 2018.

[5] D. R. Whiting, L. Guariguata, C. Weil, and J. Shaw, "IDF Diabetes Atlas: Global estimates of the prevalence of diabetes for 2011 and 2030," Diabetes Res. Clin. Pract., vol. 94, no. 3, pp. 311-321, 2011.

[6] M. B. Gomes et al., "Economic status and clinical care in young type 1 diabetes patients: A nationwide multicenter study in Brazil," Acta Diabetol., vol. 50, no. 5, pp. 743-752, 2013.

[7] M. B. Gomes et al., "Prevalence of adults with type 1 diabetes who meet the goals of care in daily clinical practice: A nationwide multicenter study in Brazil," Diabetes Res. Clin. Pract., vol. 97, no. 1, pp. 63-70, 2012.

[8] D. SBD, “Atualização sobre hemoglobina glicada : manifestações clínicas," pp. 361-369, 2015.

[9] Diabetes Control and Complications Trial Research Group et al., "The Effect of Intensive Treatment of Diabetes on the Development and Progression of Long-Term Complications in Insulin-Dependent Diabetes Mellitus," N. Engl. J. Med., vol. 329, no. 14, pp. 977-986, Sep. 1993.

[10] S. R. Johnson, M. N. Cooper, T. W. Jones, and E. A. Davis, "Long-term outcome of insulin pump therapy in children with type 1 diabetes assessed in a large population-based case-control study," Diabetologia, vol. 56, no. 11, pp. 23922400, 2013.

[11] "Equipamentos Veterinários, Equipamentos Médicos - Delta Life - Tecnologia a serviço da vida." [Online]. Available: https://www.deltalife.com.br/. [Accessed: 24-May-2019].

[12] L. E. G. Martins, H. De Faria, T. Cunha, T. De Oliveira, D. E. Casarini, and J. A. Colucci, "Development of a LowCost Insulin Infusion Pump : Lessons Learned from an Industry Case," pp. 1-6, 2015.

[13] L. E. G. Martins and T. Gorschek, "Requirements engineering for safety-critical systems: A systematic literature review," Inf. Softw. Technol., vol. 75, pp. 71-89, 2016. 